\title{
Defunding of Diversity and Inclusion Programs in Undergraduate and Graduate Medical Education
}

\author{
Lakshmi Karra, MD, MS | Melissa Johnson, MD | Cleveland Piggott, MD, MPH
}

PRIMER. 2020;4:11.

Published: 6/23/2020 | DOI: 10.22454/PRiMER.2020.971896

\section{To the Editor:}

The COVID-19 pandemic has amplified long-existing health disparities and demonstrated that communities of color are disproportionately affected-a phenomenon that is not unique to this pandemic. In light of these extreme health disparities, it is crucial to continue to fund programs that support health equity in our communities and diversify the physician workforce. As medical professionals and educators, we are concerned about the financial implications of the COVID-19 pandemic on the future of these undergraduate and graduate medical education programs.

The Society of Teachers of Family Medicine (STFM) and other family medicine organizations have been vocal about these principles, encouraging and promoting programs that increase physician diversity and include education on disparities during undergraduate and graduate medical training as stated in the STFM's Underrepresented in Medicine (URM) Initiative ${ }^{1}$ and Health Equity Goals. ${ }^{2}$ There is ample research demonstrating the positive impact of having a physician that looks like you, not just for an individual's health outcomes, but for the health outcomes of an entire community. ${ }^{3}$ Educating physicians to be aware of implicit bias, systematic oppression, and structural inequities is an equally vital part of creating a physician workforce that can address and improve health disparities.

Furthermore, investment in initiatives at the institutional level are crucial to ensure a culture of progress that fosters individual training as well as organizational change. These initiatives include pipeline programs, offices of diversity and inclusion, and programs that promote and support staff and faculty of color. ${ }^{4}$ They also include curriculum development and delivery to ensure that all physicians, regardless of race, understand, consider, and address social determinants of health in their advocacy efforts and clinical practice. Importantly, advocacy efforts and conversations about the social determinants of health are incomplete if we do not explicitly call out racism and its systemic effects on our society and medicine-a fact that has become clearer to many medical professionals in light of recent high-profile instances of racial injustice.

The financial impact of the COVID-19 pandemic is significant, unprecedented, and will undoubtedly affect all levels of physician training, recruitment, and promotion. However, the impacts of cuts to diversity funding and pipeline programs will be significant, not just at this point in time, but for a generation of physicians and patients to come. We are already beginning to see the effects. Here in Colorado, a 7-year program linking the community to our medical campus was completely defunded. At a time when health inequities are exposed and highlighted, it would be short-sighted to slow our progress. Rather, it is an opportunity to commit to our efforts to improve physician diversity, community engagement, and fight for health equity. While we hope for an end to this pandemic, we know there will be another health care crisis around the corner-one that will continue to disproportionately and unjustly affect communities of color, unless we stay the course and continue to strive for physician diversity and fund programing that continues to address inequity and racism. 


\section{Corresponding Author}

Lakshmi Karra, MD, MS

University of Colorado Family Medicine Residency, 1001 Yosemite St, Denver, C0 80203. 303-602-4545. Fax: 303-602-4550.

lakshmi.karra@cuanschutz.edu

\section{Author Affiliations}

Lakshmi Karra, MD, MS - University of Colorado Family Medicine Residency

Melissa Johnson, MD - Undergraduate Medical Education Team for the University of Colorado Department of Family Medicine

Cleveland Piggott, MD, MPH - University of Colorado Department of Family Medicine Department of Diversity and Health Equity, and University of Colorado Department of Family Medicine Undergraduate Medical Education Team

\section{References}

1. Walters E. Building a diverse academic family medicine workforce: URM initiative focuses on four strategic areas. Ann Fam Med. 2020;18(1):87-88. https://doi.org/10.1370/afm.2511

2. Society of Teachers of Family Medicine. Key Initiatives: Health Equity. https://www.stfm.org/about /keyinitiatives/healthequity/. Accessed May 13, 2020.

3. Marrast LM, Zallman L, Woolhandler S, Bor DH, McCormick D. Minority physicians' role in the care of underserved patients: diversifying the physician workforce may be key in addressing health disparities. JAMA Intern Med. 2014;174(2):289-291. https://doi.org/10.1001/jamainternmed.2013.12756

4. Rodríguez JE, Campbell KM, Mouratidis RW. Where are the rest of us? Improving representation of minority faculty in academic medicine. South Med J. 2014;107(12):739-744. https://doi.org/10.14423 /SMJ.0000000000000204

Copyright $\odot 2020$ by the Society of Teachers of Family Medicine 